Abstract

\title{
Does the Cytoplasmic Tail Matter? Mechanism of Viral Envelope Glycoprotein Targeting by Membrane-Associated-RING-CH (MARCH) Proteins ${ }^{+}$
}

\author{
Cheng man Lun, Abdul A. Waheed and Eric O. Freed* \\ Virus-Cell Interaction Section, HIV Dynamics and Replication Program, NCI-Frederick, Frederick, MD 21702, \\ USA; chengman.lun@nih.gov(C.M.L.); waheedab@nih.gov(A.W.) \\ * Correspondence: efreed@mail.nih.gov \\ † Presented at Viruses 2020-Novel Concepts in Virology, Barcelona, Spain, 5-7 February 2020. \\ Published: 8 June 2020
}

\begin{abstract}
The MARCH family of RING-finger E3 ubiquitin ligases comprise 11 members that have been reported to play a variety of roles in the downregulation of cell-surface proteins involved in adaptive immunity. The RING-CH domain of MARCH proteins is thought to ubiquitinate the cytoplasmic tails (CTs) of target proteins, leading to protein degradation through either lysosomal or proteasomal pathways. Three MARCH proteins (MARCH1, 2, and 8) have recently been reported to target the HIV-1 envelope glycoprotein (Env) and vesicular stomatitis virus G glycoprotein (VSV-G), thereby impairing the infectivity of HIV-1 virions bearing HIV-1 Env or VSV-G. However, the mechanism of antiviral activity remains poorly defined. Our data show that MARCH proteins antagonize the full-length forms of HIV-1 Env, VSV-G, and Ebola glycoprotein (GP), and impair the infectivity of HIV-1 virions bearing these viral glycoproteins. This Env-targeting activity of the MARCH proteins requires the E3 ubiquitin ligase activity of the RING-CH domain. We observe that the MARCH protein targeting of VSV-G is, to a large extent, CT-dependent. In striking contrast, the MARCH-protein targeting of HIV-1 Env and Ebola GP does not require the CT. Confocal microscopy data demonstrate that MARCH proteins are able to trap the viral glycoproteins in an intracellular compartment. We observe that the endogenous expression of MARCH8 in T-cell lines and PBMCs is inducible by type I interferons ( $a$ and $b$ ) and is also upregulated by HIV-1 infection. Current studies are aimed at identifying the cellular target for MARCH-mediated ubiquitination in the context of their antiviral activity. These results will clarify the mechanism by which MARCH proteins antagonize viral glycoproteins and provide insights into the antiviral role of cellular inhibitory factors in Env biogenesis, trafficking, and virion incorporation.
\end{abstract}

Keywords: MARCH proteins; E3 ubiquitin ligase; viral glycoproteins; cytoplasmic tail; antiviral factor

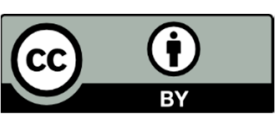

(C) 2020 by the authors. Licensee MDPI, Basel, Switzerland. This article is an open access article distributed under the terms and conditions of the Creative Commons Attribution (CC BY) license (http://creativecommons.org/licenses/by/4.0/). 